
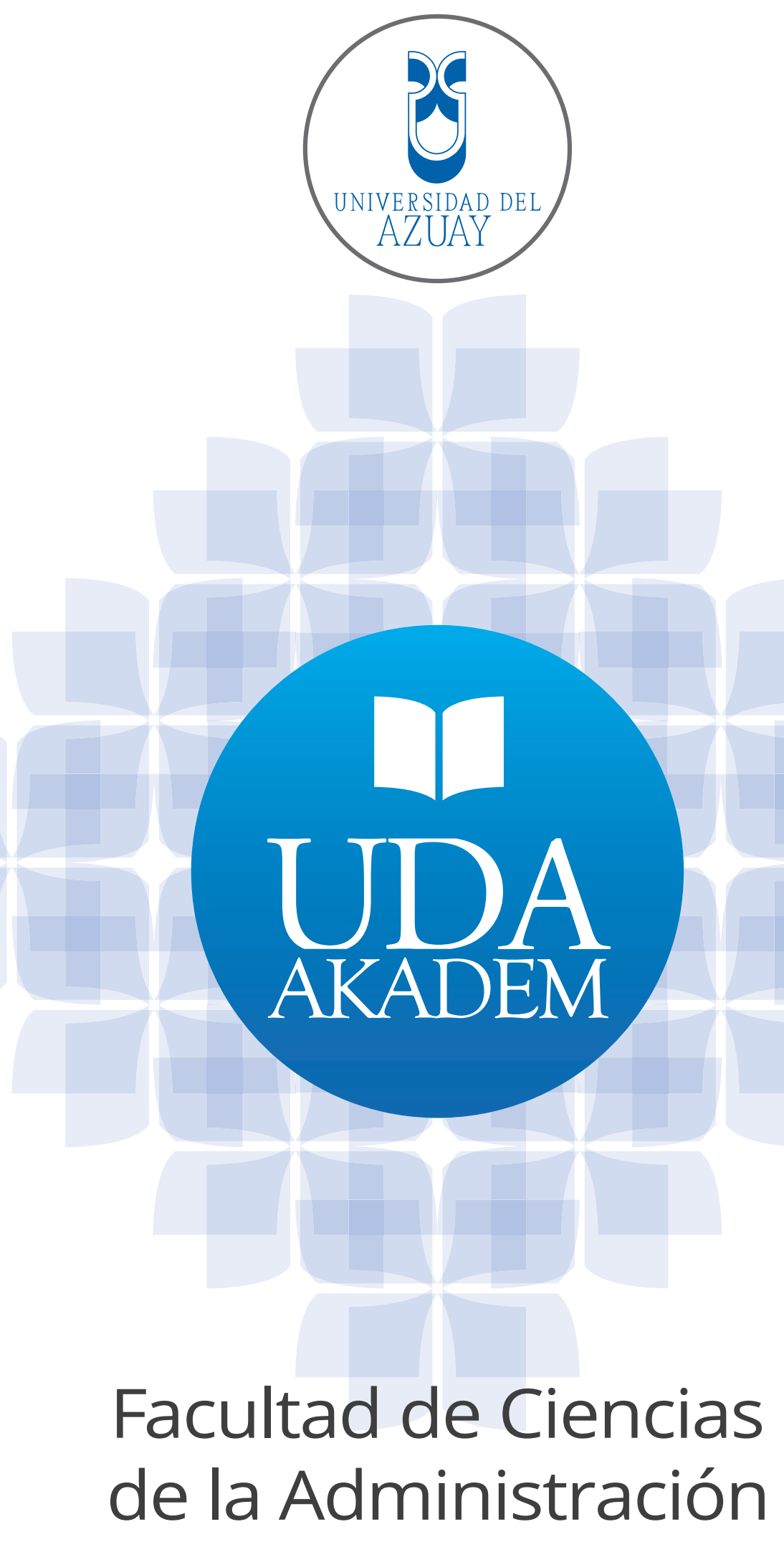

Número 


\section{LA EVOLUCIÓN DE LA URBE HACIA LAS CIUDADES INTELIGENTES}

Lenin Xavier Erazo Garzón

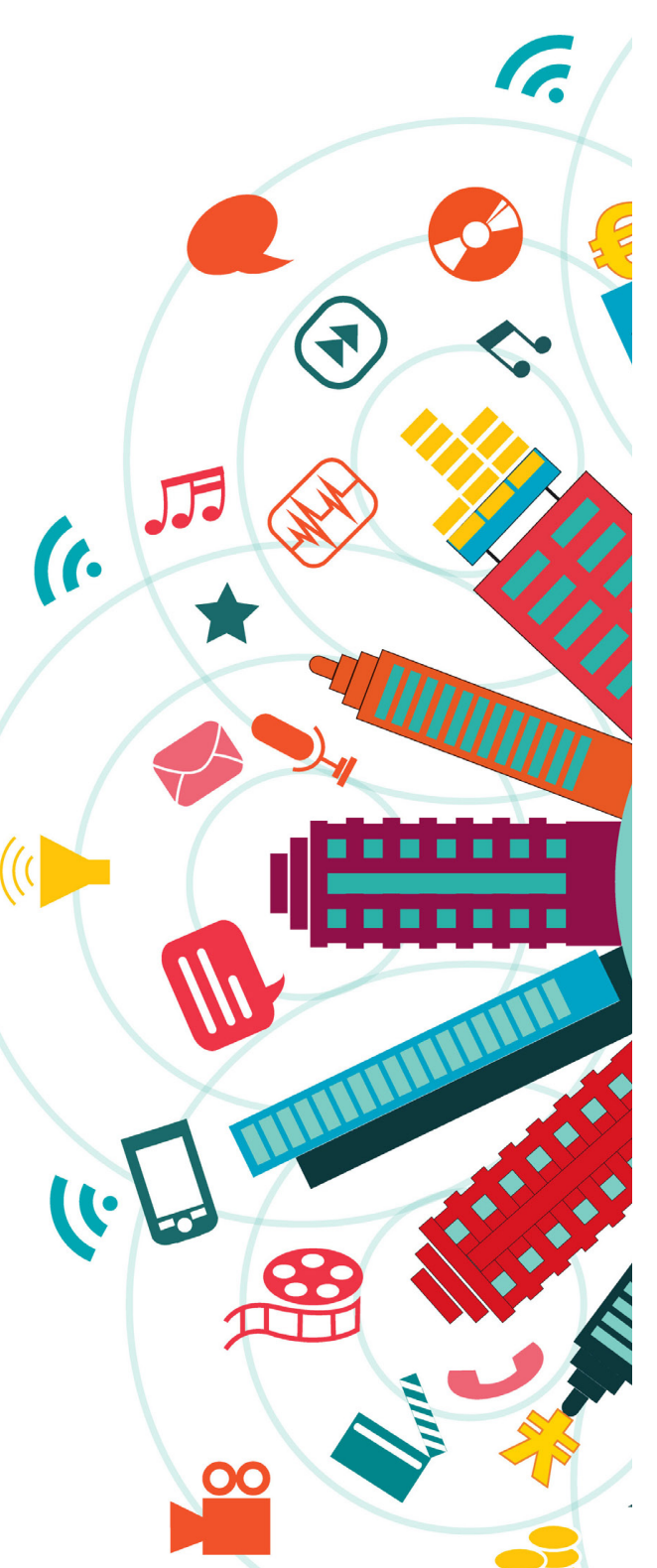

\section{Resumen}

En el siglo XXI, debido a la creciente concentración de la población en los núcleos urbanos, las ciudades se enfrentan a grandes retos de sostenibilidad de la sociedad a largo plazo, a fin de convertirse en verdaderos polos de desarrollo económico, social y cultural.

Como respuesta a esta problemática, las iniciativas estratégicas de ciudad inteligente (Smart City) se han expandido por todo el mundo y el Ecuador no ha sido la excepción. Tanto el gobierno central como los gobiernos seccionales están emprendiendo importantes proyectos en el marco de la ciudad inteligente; sin embargo, dentro de la sociedad ecuatoriana, este concepto aún resulta poco conocido y familiar.

Frente a esta circunstancia, este trabajo de investigación tiene como propósito clarificar la visión del concepto de ciudad inteligente, mediante una revisión bibliográfica de la literatura científica y comercial, que responde a las siguientes interrogantes: ¿Qué es una ciudad inteligente? ¿Es un concepto que ha surgido recientemente? y de no ser así ¿Cómo ha evolucionado este concepto? ¿Qué áreas o sistemas del entorno urbano abarca el concepto de ciudad inteligente? ¿Qué servicios puede prestar? entre las más importantes.

Palabras clave: ciudad inteligente, ciudad ubicua, entorno urbano, inteligencia urbana, internet de las cosas, sensores, domótica

\section{Abstract}

Due to the increasing concentration of population in urban areas in the XXI century, cities are facing major sustainable challenges of society in the long term so as to become real centers of economic, social and cultural development. In response to this problem, strategic initiatives of a Smart City have expanded worldwide, and Ecuador is not an exception. Both the central government and regional governments are undertaking major projects in the framework of the smart city; however, this concept is still little known and unfamiliar within the Ecuadorian society. Given this circumstance, this research paper aims to clarify the perspective of the smart city concept through a bibliographic review of the scientific and commercial literature, which answers the following questions among the most important ones: What is a smart city? Is it a concept that has emerged recently? And if not, how this concept has evolved? What areas or systems of the urban environment encompass the concept of smart city? What services can it provide?

Keywords: Smart City, Ubiquitous City, Urban Environment, Urban Intelligence, Internet of Things, Sensors, Home Automation 


\section{Introducción}

A finales del siglo XX se comenzó a experimentar por primera vez en la historia de la humanidad, el fenómeno de la urbanización, que implica una progresiva migración de las zonas rurales hacia las ciudades, lo que convierte estos entornos urbanos en potenciales oportunidades de experimentación cultural, social y económica; sin embargo, con la concentración de grandes grupos humanos en las ciudades han surgido nuevos problemas por resolver en el siglo XXI, tales como la congestión del tráfico, la baja cobertura en el abastecimiento de servicios, el incremento de la inseguridad, la polución del aire, entre otros (Sheltona, Zookb, \& Wiigc, 2014).

En la actualidad, aproximadamente el 54\% de la población mundial vive en las ciudades, frente al 30\% que lo hacía en 1950, y se estima que para el 2050 alcance el 66\% (United Nations, Department of Economic and Social Affairs, Population Division, 2014).

Con estos antecedentes, el siglo XXI está llamado a ser el siglo de las ciudades, y la ciudad inteligente (Smart City) emerge como una gran oportunidad para la gestión eficiente y eficaz de los entornos urbanos, con el fin de contribuir positivamente a mejorar la competitividad y el desarrollo sostenible de las ciudades; fomentar y garantizar una gobernabilidad transparente, participativa e inclusiva; y en definitiva mejorar la calidad de vida de los ciudadanos (AMETIC - Foro TIC para la sostenibilidad, 2012).

Particularmente en el Ecuador se observa cómo el gobierno central y los gobiernos seccionales están enmarcando sus políticas, planes, programas y proyectos en torno al concepto de ciudad inteligente (IEEE Smart Cities Initiative, 2015), incluso en sus campañas publicitarias están calificando a las ciudades con el término "inteligente". Asimismo recientemente en el mes de junio, se realizó en la ciudad de Cuenca el I Seminario Internacional Smart Cities, organizado por el Colegio de Ingenieros Eléctricos y Electrónicos del Azuay (CIEELA); con el auspicio de las Universidades de Cuenca (UDC), del Azuay (UDA) y Católica de Cuenca (UCACUE), lo que demuestra un gran interés por su desarrollo en la región (CIEELA, 2015). Sin embargo, pese a estas iniciativas el concepto ciudad inteligente aún resulta poco conocido y familiar. Existen una serie de interrogantes tales como: ¿Qué es una ciudad inteligente? ¿Es un concepto que ha surgido recientemente? y de no ser así ¿Cómo ha evolucionado este concepto? ¿Qué áreas o sistemas del entorno urbano abarca el concepto de ciudad inteligente? ¿Qué servicios puede prestar? entre las más importantes.

En este sentido el presente artículo es una revisión bibliográfica que pretende dar respuesta a las mencionadas interrogantes. 


\section{Conceptualización de ciudad inteligente}

La ciudad inteligente es un concepto amplio, emergente y dinámico, dada la cantidad de sistemas con los que se asocia en el entorno urbano (sistemas de movilidad, comunicación, hídricos, energéticos, recolección de desechos, seguridad, entre otros). Es así que para el concepto de ciudad inteligente existen varias definiciones; pero ninguna ha sido universalmente aceptada como un estándar. En la tabla 1 se presentan las definiciones más recurrentes dentro de la literatura académica y comercial.

\begin{tabular}{|c|c|}
\hline Definición & Referencia \\
\hline $\begin{array}{l}\text { "Smart City es una ciudad basada en seis características (economía, } \\
\text { personas, gobierno, movilidad, ambiente y vivienda) y en la combinación } \\
\text { "inteligente" de ciudadanos independientes, decisivos y conscientes." }\end{array}$ & (Giffinger, y otros, 2007) \\
\hline $\begin{array}{l}\text { "Una ciudad es inteligente cuando las inversiones en capital social y } \\
\text { humano, y la infraestructura de la comunicación tradicional (transporte) y } \\
\text { moderna (tecnologías de información y comunicación) incentivan el cre- } \\
\text { cimiento económico sostenible y una mejor calidad de vida, con un sabio } \\
\text { uso de los recursos naturales, a través de un gobierno participativo." }\end{array}$ & (Caragliu, Del Bo, \& Nijkamp, 2011) \\
\hline $\begin{array}{l}\text { "Smart City es un entorno urbano que utiliza las tecnologías de informa- } \\
\text { ción para sentir, analizar, integrar y reaccionar con inteligencia en las ac- } \\
\text { tividades que se realizan en la ciudad, en sus empresas, infraestructuras, } \\
\text { servicios, entorno ambiental y especialmente con sus ciudadanos, convir- } \\
\text { tiéndose así en un lugar mejor para vivir, trabajar y hacer negocios." }\end{array}$ & (IBM, 2010) \\
\hline $\begin{array}{l}\text { "Smart City es el producto de ciudad digital combinado con la Internet de } \\
\text { las cosas." }\end{array}$ & (Su, Li, \& Fu, 2011) \\
\hline $\begin{array}{l}\text { "Smart City es una ciudad que controla e integra toda su infraestructura } \\
\text { crítica: carreteras, puentes, túneles, rieles, metros, aeropuertos, puertos } \\
\text { marítimos, comunicaciones, redes de agua y energía, e incluso los edifi- } \\
\text { cios principales, para así optimizar sus recursos, planificar sus activida- } \\
\text { des de mantenimiento preventivo, supervisar los aspectos de seguridad y } \\
\text { mejorar los servicios de sus ciudadanos." }\end{array}$ & (Hall, 2000) \\
\hline $\begin{array}{l}\text { "Smart City es una ciudad en la que se puede combinar tecnologías tan } \\
\text { diversas como el reciclaje de agua, redes de energía avanzada y las co- } \\
\text { municaciones móviles con el fin de reducir el impacto ambiental y ofrecer } \\
\text { a sus ciudadanos una mejor calidad de vida." }\end{array}$ & (Setis-Eu , 2012) \\
\hline $\begin{array}{l}\text { "Smart City es aquella ciudad que usa las tecnologías de la información y } \\
\text { las comunicaciones para hacer que tanto su infraestructura crítica, como } \\
\text { sus componentes y servicios públicos ofrecidos sean más interactivos, } \\
\text { eficientes y los ciudadanos puedan ser más conscientes de ellos." }\end{array}$ & (Telefónica, 2011) \\
\hline
\end{tabular}

Como resultado del análisis de las definiciones antes citadas, se puede destacar lo siguiente:

- $\quad$ En su mayoría las definiciones coinciden en el uso intensivo de las tecnologías de información y comunicación (TIC) como fuerza facilitadora para que una ciudad adquiera una cierta característica de "inteligencia". A su vez, dan a entender que una ciudad para que sea considerada verdaderamente "inteligente" debe partir de una visión holística de sus necesidades, involucrando a múltiples participantes: ciudadanos, gobierno, academia y empresa privada, a fin de desarrollar soluciones basadas en TIC que integren e interconecten todos los sistemas que componen el entorno urbano. 
- Otra fuerza facilitadora de la ciudad inteligente es la inversión en capital humano y social, debido a que un entorno urbano que cuente con ciudadanos competentes, especialmente en el uso de las TIC, se convierte en una fuente generadora de desarrollo y de ventajas competitivas sostenibles.

- Tomando como base la definición de Su, Li y Fu, el concepto de ciudad inteligente desde una visión integral es la conjunción de todas las iniciativas estratégicas que se han implementado en las últimas décadas, bajo los conceptos de ciudad del conocimiento, ciudad digital, ciudad ubicua, ciudad sustentable, entre otros. Asimismo, la combinación con el concepto de "Internet de las cosas", es una visión futurista en el que no solo cada vez más personas podrán estar interconectadas, sino que también podrán interconectarse todos los dispositivos y objetos del entorno urbano que deban gestionarse o controlarse (redes eléctricas y de agua potables, sistemas de transporte, edificios, vehículos, electrodomésticos, ropa, etc.). Al incorporar las TIC a estos elementos, adquieren una característica de "inteligencia", que les permitirá intercambiar información entre ellos y tomar decisiones en forma autónoma ante las condiciones cambiantes del entorno urbano, en procura de mejorar su gestión.

- Sin lugar a dudas todas las definiciones de ciudad inteligente apuntan a maximizar la economía y el bienestar de las ciudades, en base a una gestión tecnológica efectiva de los servicios urbanos y recursos naturales.

Como se ha mencionado el concepto de ciudad inteligente engloba iniciativas para todos los sistemas del entorno urbano, las cuales pueden ser clasificadas dentro de seis dimensiones o características de "inteligencia", según el informe "Smart Cities. Ranking of European medium-sized cities" (Giffinger, y otros, 2007):

- Smart Economy: ciudades que implementan modelos de negocios innovadores para el sector público y privado mediante el uso intensivo de las TIC con el propósito de alcanzar un desarrollo económico sostenible.

- Smart People: ciudades que cuentan con ciudadanos (capital humano) formado y capacitado, especialmente en el campo de las TIC.

- Smart Governance: ciudades cuyo gobierno utiliza ampliamente las TIC para ofrecer servicios públicos y sociales de calidad, fomentar la participación ciudadana y la trasparencia.

- Smart Environment: ciudades que utilizan las TIC para incidir en la eficiencia energética y la reducción del impacto ambiental.

- Smart Living: ciudades que cuentan con políticas, normas y planes que apoyan el uso de las TIC para incidir en la cohesión social, el fomento de la cultura, la sosteniblidad de la planificación 
urbanística, la calidad y cobertura de los servicios urbanos, la seguridad ciudadana, la mejora de las condiciones de salud y el turismo atractivo.

- Smart Mobility: ciudades que utilizan las TIC para mejorar la movilidad, el tráfico y la logística en la ciudad.

Si bien se han clasificado las iniciativas de una ciudad inteligente en varias dimensiones, solo alcanzan todo su potencial cuando integran varios sistemas de una ciudad, a fin de intercambiar información y proporcionar una visión conjunta de gestión urbana. Como ejemplo, existen soluciones integrales que ayudan a gestionar el tráfico en tiempo real de una ciudad, y que al mismo tiempo, alimentan a los sistemas de búsqueda de estacionamientos o ayudan a identificar zonas de la ciudad con elevados niveles de contaminación.

Los aspectos socio-económicos, culturales, el compromiso con el ambiente y la importancia que se otorgue a la infraestructura y los servicios en una región geográfica, determinan las terminologías que se utilicen para referirse a la "inteligencia" de la ciudades; es así que en Norteamérica las más utilizadas son: Intelligent City, Intelligent Community y Digital Community; en Europa Smart City y Eco City; y, en Asia Smart City, Digital City y Ubiquitous City. En Norteamérica la orientación principal de ciudad inteligente es hacia fomentar el desarrollo socioeconómico y comunitario en base a la colaboración entre gobierno, empresa y sociedad. En Europa, a su vez, el concepto se orienta a la infraestructura y sustentabilidad para aprovechar en forma efectiva los recursos y mejorar la calidad de vida de los ciudadanos. Por último, en Asia se orienta a la sociedad en base al desarrollo de talento humano especializado en nuevas tecnologías y la entrega de servicios de calidad (Gobierno de la Ciudad de Buenos Aires, 2012).

\section{Evolución de la inteligencia urbana}

El continúo avance de la ciencia y la tecnología ha dado lugar a la evolución de la inteligencia urbana; en sus inicios las ciudades eran simples estructuras físicas cuyas funciones fueron proporcionar refugio y protección; los habitantes tenían una movilidad limitada y las comunicaciones y transacciones mercantiles se realizaban cara a cara. Con la llegada de la industrialización se construyeron redes de suministro de agua y energía, redes de eliminación de residuos líquidos, redes de transporte y se ampliaron los canales de distribución, proveyendo de servicios y productos básicos a la ciudadanía. En la segunda mitad del siglo XIX, las ciudades comenzaron con la construcción de los sistemas de comunicación, como: el telégrafo, el teléfono y la radio. Con la llegada de la era digital, los elementos de la inteligencia urbana fueron apareciendo progresivamente y dando origen a la llamada convergencia tecnológica. Primero, a finales de la década de 1960, con la introducción de las telecomunicaciones digitales, se expandió notablemente la conectividad hasta llegar hoy en día a una escala global, mediante internet y las redes de telefonía móvil. Después la reducción del tamaño y el aumento del rendimiento de los aparatos semiconductores permitieron el desarrollo de varios productos; a mitad de la década de 1980 llegaron los ordenadores personales de escritorio, 
luego aparecieron los ordenadores portátiles y los dispositivos móviles, los cuales se interconectaron a través de las redes digitales; a continuación los pequeños microprocesadores se integraron en los artefactos de uso cotidiano desde automóviles hasta cámaras digitales. Finalmente la presencia de los sensores e identificadores digitales y su integración a los artefactos posibilitó que se pueda tomar decisiones y actuar rápidamente ante ciertos estados físicos, por ejemplo, los GPS permiten a los automóviles y teléfonos móviles determinar su ubicación (Mitchell, 2007).

Ahora bien, el software se ha convertido en un integrador de todos los elementos de inteligencia en un sistema a gran escala, coordinado y distribuido geográficamente. Todo esto ha dado lugar a una tecnología de inteligencia integrada de forma ubicua en todos los entornos urbanos.

El concepto de ciudad inteligente ha sido empleado desde hace más de veinte años y ha evolucionado en base a los diversos sectores en los que ha intervenido dentro del entorno urbano. En su inicio el sector fundamental fue la eficiencia energética y la reducción de las emisiones de carbono, hasta incursionar en un campo más amplio como es el empleo de las TIC en la gestión del gobierno, los servicios públicos y la infraestructura de la ciudad.

Cocchia en su trabajo de revisión sistemática de la literatura manifiesta que el primer estudio sobre Smart City fue publicado en el año 1994, y que entre 1994 y 1997 no ha encontrado más publicaciones sobre esta temática. A partir de 1997 el número total de publicaciones ha crecido a un ritmo estable hasta 2010. Desde 2010 su crecimiento se ha duplicado año tras año (Cocchia, 2014). La figura 1 muestra la tendencia de publicación de artículos científicos relacionado con Smart City, incluyendo publicaciones de Digital City ya que en los últimos años se ha convertido en una sub-área de Smart City.
Figura: 1 Tendencia de artículos científicos relacionados con Smart City

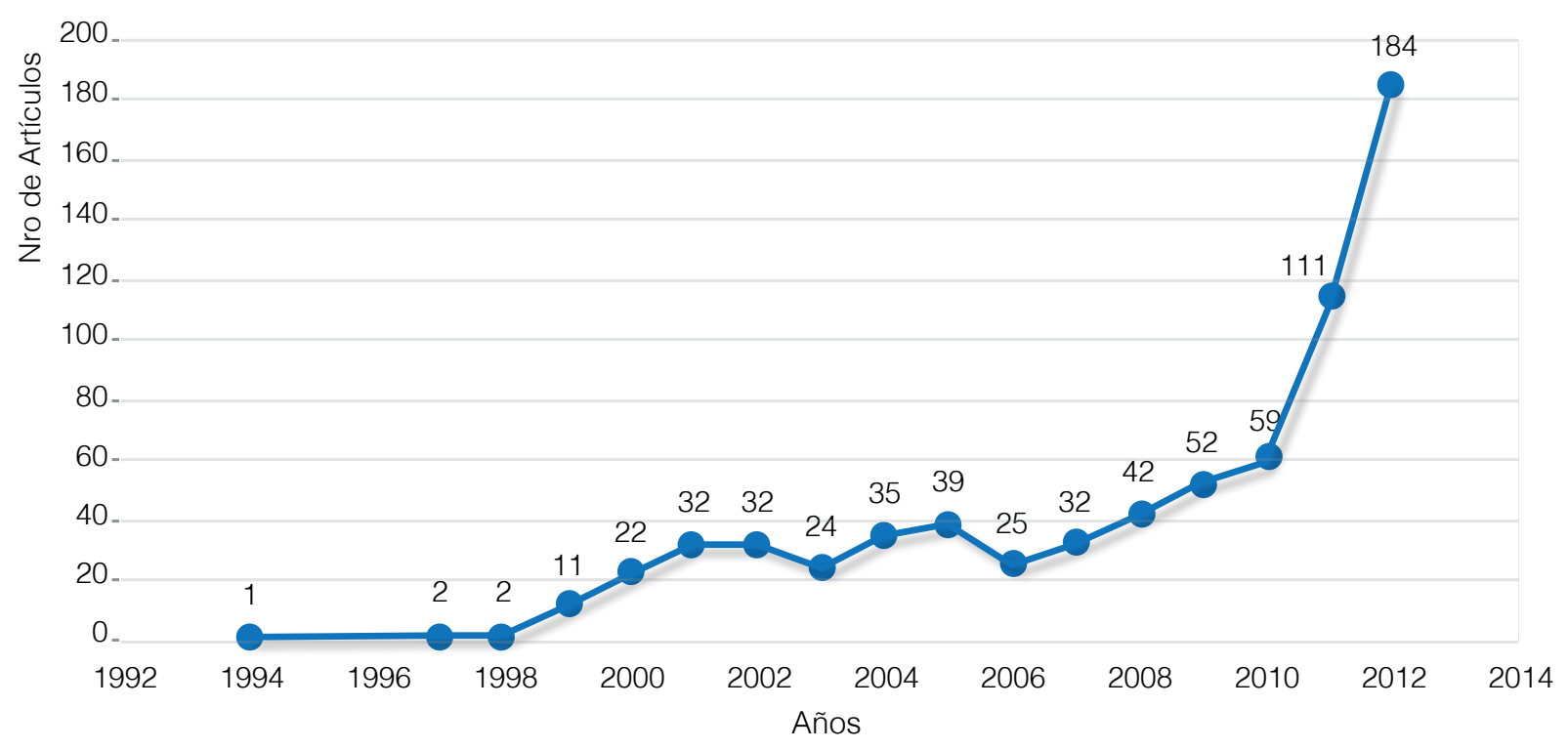


Los eventos que en los últimos 20 años han influido e impulsado el desarrollo científico del concepto de ciudad inteligente probablemente son los siguientes:

1. En 1997 la firma del protocolo de Kyoto cuyo propósito fue establecer límites y reducir las emisiones de los gases de efecto invernadero.

2. En 2000 la expansión de la internet en todos los entornos de la vida cotidiana. Los avances en el área de las TIC, internet de banda ancha, dispositivos móviles, redes de sensores inteligentes, aplicaciones web y móviles, plataformas abiertas, etc., han contribuido a la implementación de servicios electrónicos e inteligentes en diversas áreas del convivir humano, principalmente por parte del gobierno central y local. (Ishida, 2000)

3. El 16 de febrero de 2005 entró en vigor el protocolo de Kyoto y las iniciativas estratégicas relacionadas con Smart City y la protección del ambiente se incrementaron en todo el mundo.

4. En 2007 la compañía Apple, lanzó al mercado el primer teléfono inteligente llamado i-phone, como resultado de la convergencia tecnológica que combina las redes de datos de alta velocidad (Wi$\mathrm{Fi}$, banda ancha móvil) y software a través de aplicaciones móviles para proporcionar servicios electrónicos en tiempo real a los ciudadanos. (Giffinger, y otros, 2007) A partir de este momento, el adjetivo "inteligente" se comenzó a asociar con varios dispositivos o artefactos de uso cotidiano de las personas (refrigeradora, televisión, etc.).

5. En 2008 IBM fue la primera compañía que lanzó la iniciativa de "Smart Planet" para fomentar en los líderes de negocio, el gobierno y la sociedad civil la importancia de dotar de inteligencia a los sistemas y procesos que hacen que el mundo funcione, cosas que nadie reconocería como sistemas informáticos: vehículos, electrodomésticos, carreteras, redes eléctricas, ropa, etc. El objetivo es conseguir que estos sistemas funcionen de forma más instrumentada, interconectada e inteligente haciendo uso de las propias tecnologías de IBM, tales como: "cloud computing", "analytics", "mobile technology" y "social bussiness" (IBM, 2015). A raíz de esta iniciativa, otras empresas de tecnologías han incursionado en esta área, tales como: Cisco, ABB, HP, Siemens, Ericsson, etc.

6. En 2010 la Unión Europea puso en marcha el Plan "Europe 2020 Estrategy" cuyos objetivos estratégicos son: desarrollo inteligente con inversión en áreas de la educación, investigación e innovación, desarrollo sostenible invirtiendo en tecnologías y recursos más limpios y desarrollo inclusivo con fuerte énfasis en la creación de empleo y reducción de la pobreza. Para alcanzar los objetivos citados cada uno de los países de Europa debe diseñar y ejecutar iniciativas estratégicas en el marco de Smart City. (Setis-Eu, 2015). Este evento ha influido en gran medida para la creación y publicación de artículos científicos sobre Smart City, convirtiéndose en una tendencia política impulsada por varios organismos internacionales. 
A su vez se espera que en los próximos años exista un fuerte compromiso para el desarrollo de ciudades cada vez más sostenibles mediante el empleo de las TIC, debido a que en septiembre de 2015, los Estados miembros de la Organización de Naciones Unidas aprobaron la Agenda 2030 para el desarrollo sostenible, que incluye el objetivo número 11: "Ciudades y comunidades sostenibles", que entre los puntos más importantes hace referencia a: mejorar la planificación y gestión urbana de manera que sea participativa e inclusiva, garantizar el acceso a viviendas seguras y asequibles, crear áreas públicas verdes, mejorar los asentamientos marginales y dotar de infraestructura adecuada de transporte público (ONU, 2015).

\section{Desarrollo de iniciativas de ciudad inteligente por área geográfica}

A continuación se enlistan las áreas geográficas que mayor interés han demostrado en la implementación de estrategias de ciudad inteligente. Su determinación se ha basado en 162 artículos científicos sobre estudios de casos empíricos de Smart City, encontrados entre los años de 1994 y 2012 (Cocchia, 2014).

1. Asia es el continente con mayor número de ciudades que han implementado iniciativas de Smart City; se han identificado 40 ciudades, que representan un $43 \%$.

2. Europa, después de Asia, es el continente que posee un número relevante de ciudades, se han encontrado 32 ciudades, que representan un 34\%.

3. América del Norte es la tercera región geográfica, con 11 ciudades, que representan el $12 \%$.

4. Oceanía, Centro/Sur América y África, son los continentes que presentan menor número de ciudades inteligentes, 4, 5 y 2 ciudades respectivamente.

Las ciudades de Centro y Sur América que evidencian estudios de casos empíricos de Smart City son: Juárez y Ciudad de México (México), Nassau (Bahamas), Paraná y Río de Jainero (Brasil), lo que demuestra escaso interés e incipientes iniciativas por parte de Latinoamérica. Sin embargo, al mismo tiempo se vuelve una gran oportunidad para generar líneas de investigación en el marco de las ciudades inteligentes, con la finalidad de crear soluciones tecnológicas innovadoras que se adapten a las necesidades, idiosincrasia, aspectos socioeconómicos, culturales y educativos, y capacidades de infraestructura propias de las ciudades Latinoamericanas.

En la actualidad conocemos que a este proceso de ciudades inteligentes se han incorporado también ciudades como: Guadalajara (México), Bogotá y Medellín (Colombia), Montevideo (Uruguay) y Curitiba (Brasil), entre las más importantes (Estévez, 2015). 


\section{Servicios de una ciudad inteligente}

Con el propósito de clarificar el alcance del concepto de ciudad inteligente, en este apartado se presentan varios ejemplos de sistemas y servicios que pueden formar parte de un plan integral de ciudad inteligente.

\section{Movilidad urbana}

Uno de los problemas más agobiantes es la congestión del tráfico, con efectos negativos en las ciudades por la disminución de la productividad, la contaminación del aire y los niveles elevados de ruido. En este sentido las soluciones de Smart City se basan en el intercambio de información del tráfico en tiempo real, entre vehículos y de estos con la infraestructura urbana, a fin de mejorar la sincronización de los semáforos, la señalización, la gestión de estacionamientos, incidentes (zonas con obras) y urgencias (ambulancias, policía, bomberos); así como también recomendar rutas óptimas. Otro tipo de aplicaciones ayudan a gestionar las redes de transporte público, optimizan el uso de su infraestructura y recursos, y facilitan su mantenimiento proactivo; gracias a indicadores de uso obtenidos en tiempo real. A su vez, el vehículo eléctrico se proyecta como una solución limpia, compacta y eficiente desde el punto de vista energético.

\section{Gestión de infraestructura y equipamiento urbano}

En este contexto el objetivo primordial es optimizar el consumo de energía y agua de los edificios y viviendas, utilizando sistemas inteligentes (domótica) basados en sensores para gestionar de manera eficiente la iluminación, calefacción, aire acondicionado, ascensores, refrigeración, uso de agua, entre otros. Aún más, este mismo enfoque se puede generalizar para gestionar la infraestructura y equipamiento público, por ejemplo: 1) la calibración automática de la intensidad de la iluminación del alumbrado público, mediante la inclusión de sensores de movimiento y la configuración de parámetros, adaptándose a las necesidades del lugar y la hora; 2) la detección en tiempo real de fugas en las redes de agua potable, por medio del empleo de sistemas de sensores de ruido y presión; 3) la planificación bajo demanda de rutas óptimas de recolección de residuos, a través de sistemas de sensores que determinen si los contenedores de desechos se encuentran llenos; y 4) los sistemas inteligentes de riego en parques y jardines públicos, basados en sensores que miden el nivel de humedad de la tierra.

\section{Eficiencia energética y ambiente}

Las iniciativas de Smart City pueden llevarse a cabo desde el campo de la generación de energía, mediante el empleo de energías renovables (hidráulica, eólica, fotovoltaica) que contribuyan a reducir las emisiones de gases de efecto invernadero, hasta el campo de la distribución y consumo, a través del uso de las TIC para dotar a las ciudades de redes eléctricas inteligentes denominadas Smart Grid. Este nuevo 
enfoque reemplaza la estructura tradicional de comunicación unidireccional, por un esquema bidireccional entre los puntos de generación y consumo, con el propósito de ajustar perfectamente la producción con el consumo de energía en tiempo real y reducir las pérdidas de energía. El concepto de Smart Grid hace factible implementar sistemas de facturación más sofisticados para que los usuarios del servicio puedan conocer y controlar su consumo en tiempo real. Desde una visión holística, estas iniciativas deben integrarse con los sistemas inteligentes de transporte y con los sistemas de domótica para controlar y supervisar los elementos existentes en un edificio o vivienda.

\section{Gobierno y ciudadanía}

En este campo las iniciativas se derivan del concepto de ciudad digital y tienen como propósito alcanzar un gobierno transparente, participativo e inclusivo. Mediante el uso de las TIC se ofrecen a los ciudadanos y empresas servicios online para consultar información básica, realizar trámites y pagos, plantear reclamos y preguntas, participar en encuestas y votaciones, conocer la gestión del gobierno, entre las más importantes. Otro tipo de servicio son los datos abiertos (Open Data) que fomentan la disponibilidad en forma libre de los datos sobre diferentes temáticas (geográficas, meteorológicas, económicas, etc.) para que puedan ser aprovechados por la sociedad en la construcción de nuevas ideas o productos.

\section{Comercio}

Las soluciones de Smart City implementan plataformas integradas de pago para los múltiples servicios que se ofrecen en una ciudad, tales como: sistemas de transporte, energía eléctrica, agua potable, entradas a museos y teatros, entre otros. Asimismo, ofrecen medios de pago sin necesidad de dinero físico, tales como: tarjetas recargables prepago o pago a través de móvil, lo que sin lugar a dudas repercute en la comodidad de los ciudadanos.

\section{Educación, cultura y turismo}

Las iniciativas de Smart City en esta área aprovechan la infraestructura de TIC desplegada en las ciudades para apoyar mediante medios electrónicos los procesos de enseñanza - aprendizaje (e-learning) y el trabajo colaborativo a distancia (groupware, teletrabajo). Otro tipo de iniciativas son las soluciones que utilizan dispositivos móviles, sensores de localización (GPS), mapas y realidad aumentada para proporcionar información sobre servicios culturales y turísticos. En este ámbito se debe propiciar la creación de alianzas estratégicas entre el gobierno y las organizaciones relacionadas con el turismo para crear canales únicos y homogéneos de provisión de los servicios. A manera de ejemplo se tienen los tours multimedia de museos y ciudades, que recomiendan rutas a los ciudadanos y visitantes de acuerdo con sus gustos y preferencias. 


\section{Seguridad ciudadana}

Incluyen sistemas avanzados de seguridad, integrados por centros de atención de emergencias, cámaras de videovigilancia y redes de sensores, con la posibilidad de recopilar y procesar grandes cantidades de información (imágenes, videos, etc.) en tiempo real.

\section{Salud}

Se enmarcan aquellas soluciones tecnológicas tales como la telemedicina y la teleasistencia, orientadas a mejorar la calidad de vida de las personas con capacidades especiales, enfermos y ancianos; mediante el uso de sensores de localización (GPS) y bio-sensores para monitorear a distancia su estado de salud, brindarles protección y evitar en la medida de lo posible que sean dependientes. El alcance detrás de este servicio es mucho más ambicioso, se trata de implementar un sistema integrado de salud que incluya una base de datos única de historiales clínicos, la cual sea compartida entre hospitales, clínicas, centros de salud y farmacias. La sanidad pública también es considerada en una ciudad inteligente, mediante soluciones inteligentes, que de forma proactiva, eviten posibles riesgos de salud, por ejemplo, un ciudadano al ingresar a un restaurante puede ser alertado sobre el incumplimiento de los permisos de salud para su funcionamiento.

\section{Conclusiones}

A lo largo del presente artículo se ha profundizado sobre el alcance y la evolución que ha tenido el concepto de ciudad inteligente. Es así que se ha llegado a la conclusión de que es un concepto holístico, que hace uso intensivo de las TIC para gestionar, integrar e interconectar todos los sistemas que componen un entorno urbano, de tal forma que optimicen los recursos que consumen y brinden servicios de calidad.

La evolución hacia una ciudad inteligente no es sencilla, es un camino progresivo y por etapas, ya que por la amplitud del concepto se demanda grandes cantidades de inversión y esfuerzo.

De ahí que lo fundamental es partir de una visión estratégica de ciudad inteligente en la que todas las iniciativas estén alineadas bajo un objetivo común. Esto hará viable que todos los sistemas inteligentes, creados a partir de las diversas iniciativas, puedan integrarse e intercambiar información entre ellos, y formar parte de un gran ecosistema a nivel de toda la ciudad. Solo en ese momento se hablaría de una verdadera ciudad inteligente, compuesta por millones de dispositivos inteligentes y sensores interconectados entre sí, mediante redes inalámbricas de alta velocidad, y suministrando datos del estado de los componentes de las ciudad y del comportamiento de sus ciudadanos para su procesamiento y retroalimentación en tiempo real, dando lugar a una gestión efectiva del entorno urbano basada en el conocimiento.

A su vez se ha demostrado que en Latinoamérica existen incipientes iniciativas de Smart City, por lo que es prioritaria la formulación e implementación de planes, programas y proyectos en el marco de la ciudad inteligente, en los que cooperen el gobierno, la academia y 
la empresa privada; y que indudablemente estén alineados con las necesidades, idiosincrasia, aspectos socioeconómicos, culturales y educativos, y capacidades de infraestructura propias del entorno.

\section{Referencias bibliográficas}

- $\quad$ AMETIC - Foro TIC para la sostenibilidad. (2012). Smart Cities. Madrid: AMETIC.

- $\quad$ Caragliu, A., Del Bo, C., \& Nijkamp, P. (2011). Smart cities in Europe. Journal of Urban Technology, 18(2) 65-82.

- $\quad$ CIEELA. (2015). I Seminario Internacional Smart Cities \& II Seminario Internacional Smart Grid. Obtenido de http://www.eventoscieela.com/index.html

- $\quad$ Cocchia, A. (2014). Smart and Digital City: A systematic Literature Review. Springer International Publishing.

- $\quad$ Estévez, E. (14-18 de Septiembre de 2015). Curso de Fundamentos de Gobernanza Electrónica para Ciuda des Inteligentes. Universidad Nacional de la Plata. La Plata, Argentina.

- $\quad$ Giffinger, R., Fertner, C., Kramar, H., Kalasek, R., Pichler-Milanovic, N., \& Meijers, E. (2007). Smart cities -Ran king of European medium-sized cities. Vienna University of Technology.

- $\quad$ Gobierno de la Ciudad de Buenos Aires. (2012). ¿Qué es una ciudad inteligente? Descripción del enfoque del paradigma Smart City según el Gobierno de la Ciudad de Buenos Aires.

- $\quad$ Hall, P. (2000). Creative cities and economic development. Urban Studies, 37(4), 633-649.

- $\quad$ IBM. (2010). Smarter Cities. Obtenido de http://www.ibm.com/smarterplanet/in/en/smarter_cities/overview/

- $\quad$ IBM. (2015). Smarter thinking for a smarter planet. Obtenido de http://www.ibm.com/smarterplanet/es/es/overview/ideas/?re=sph

- $\quad$ IEEE Smart Cities Initiative. (2015). IEEE Smart Cities Initiative Affiliate City Profile Guayaquil, Ecuador. Obtenido de http://smartcities.ieee.org/news-bulletin/march-2015/ieee-smart-cities-initiative-affiliate-city-profi le-guayaquil-ecuador.html

- $\quad$ Ishida, T. (2000). Understanding digital cities. Digital Cities: Experiences, Technologies and Future Perspecti ves. Springer - Verlag, 7-17.

- $\quad$ Mitchell, W. J. (2007). Ciudades Inteligentes. UOC PAPERS Revista sobre la Sociedad del Conocimiento, 3-5.

- $\quad$ ONU. (2015). Agenda 2030 para el Desarrollo Sostenible. Obtenido de http://www.undp.org/content/undp/es/ home/mdgoverview/post-2015-development-agenda.html

Setis-Eu . (2012). Obtenido de setis.ec.europa.eu/implementation/technology-roadmap/European-initiative

- $\quad$ Setis-Eu. (2015). European Initiative on Smart Cities. Obtenido de https://setis.ec.europa.eu/set-plan-imple mentation/technology-roadmaps/european-initiative-smart-cities

- $\quad$ Sheltona, T., Zookb, M., \& Wiigc, A. (2014). The 'actually existing smart city'. Cambridge Journal of Regions, Economy and Society.

- $\quad$ Su, K., Li, J., \& Fu, H. (2011). Smart city and the applications. . IEEE International Conference on Electronics, Communications and Control (ICECC), 1028 - 1031.

- $\quad$ Telefónica. (2011). Smart Cities: Un primer paso hacia la internet de las cosas. Madrid - España: Ariel.

- $\quad$ United Nations, Department of Economic and Social Affairs, Population Division. (2014). World Urbanization Prospects The 2014 Revision. New York. 